\title{
Getting to Scale: Developing a Sustainable, Collaborative, Mixed-Method Approach to Space Assessment at the University of Washington Libraries
}

\author{
Jackie Belanger, Maggie Faber, and Jenna Nobs \\ University of Washington, USA
}

\section{Introduction}

This paper details changes to the University of Washington Libraries' (UW) approach to space assessment piloted during 2016-2018. The authors discuss how they have been transforming the UW Libraries' approach to space assessment to focus on ongoing data gathering, use of mixed methods, and greater staff engagement to support the effective use of assessment results. In considering our goals for the pilot, and what we wanted to change about our previous approach to space assessment, we established a series of guiding questions:

- What does a scalable, sustainable, mixed-method approach to space assessment look like for a large, complex research library system such as the UW?

- How can we develop a programmatic approach to space assessment that enables us to understand and respond to user needs both in specific libraries and holistically across our system, and also ensure that we have data available to us for longer-term space planning?

- How might we engage libraries staff meaningfully in the assessment process in order improve our ability to take action on results?

There is a significant body of literature on library space assessment, some of which focuses on taking a programmatic, mixed-methods, and/or longitudinal approach to space assessment. ${ }^{1}$ However, there are fewer studies focusing on undertaking this kind of programmatic assessment and data gathering at scale and holistically across multiple sites or a library system. ${ }^{2}$ This paper aims to address this gap. Although this paper discusses a programmatic approach to space assessment in the context of a large research library system, the authors believe that the strategies, methods, and tools piloted in this project will be relevant to libraries of various types and sizes.

\section{Context and challenges}

The University of Washington is a large research institution and includes sixteen libraries across three campuses. ${ }^{3}$ With a growing student population and the need to provide a variety of spaces to support collaborative and individual work, the UW Libraries system (and the university generally) is experiencing severe pressure on our physical spaces. Getting additional off-site storage for collections is a critical enabling factor in moving forward with any major library renovation or capital projects, but when this will happen is unclear. As a result, we are currently striving to maximize and improve the user spaces we have. However, we also need to continue to gather robust data about how spaces are currently being used so that we will be prepared to make informed decisions when we are able to make significant changes to spaces in the future.

One of the challenges to effective space assessment in such a large system is that it can be difficult to achieve a holistic, integrated approach to assessment and improvement, rather than one rooted in individual libraries. It is obviously important for individual libraries to understand the needs of their user communities and to answer questions about specific spaces (such as the libraries' Active Learning Classrooms, located in UW's Odegaard Undergraduate Library). ${ }^{4}$ However, with many assessments happening in an ad hoc way at the local branch level, it has been difficult to "connect the dots" systemically in terms of the overall outcomes we are trying to achieve with the variety of spaces we provide and also determine which improvements are unique to a single library versus those that could be more efficiently scaled across multiple libraries. Such a large, distributed system creates opportunities to tailor spaces to a variety of user needs, but also presents the potential for unevenness of data collection or an unnecessary duplication of effort. 
It can also be challenging to get traction on results because responsibility for different aspects of space are distributed throughout the organization; stakeholders include branch head librarians, facilities staff, and staff in a newly formed department overseeing access services and branch operations across multiple locations. While our past discussions of space survey results with library staff have always been met with general support, a lack of clarity about who was responsible for improvements, or how to make changes, prevented the results from being used to their full potential. This has meant that consistent feedback over time has not always been acted upon, and many staff have expressed skepticism about assessment activities because they do not always appear to lead to visible improvements.

In light of these challenges, the authors made a number of changes to the UW Libraries space assessment program during the 2016-2018 pilot. These changes focused on four key areas:

1. Developing a mixed-methods toolkit that could provide a more complete picture of space use and needs in specific spaces and that could be scaled across our system

2. Broadening and refocusing discussions with staff to surface shared questions and foster ongoing conversations about our users' space needs

3. Creating tailored reports and visualizations to draw together data from a variety of sources to encourage taking action on results

4. Taking a more collaborative approach to data analysis and emphasizing shared decision-making in order to help staff move from results to action

The following sections explore highlights from each of these areas, followed by a discussion of what the authors learned from the pilot and next steps for operationalizing this approach.

\section{Developing a scalable mixed-method approach}

The first change we made to our program was an attempt to develop a mixed-methods strategy that could be deployed across our system. The UW Libraries has a long-standing and highly successful space survey: the In Library Use Survey, which has run every three years since $2002 .{ }^{5}$ This survey provides important data about who is using library spaces, what users are doing in the spaces, and how long they stay in a library during a specific visit. In the past, survey data has been used for improvement and advocacy, and we were scheduled to undertake another survey in 2018. The In Library Use Survey has been our primary method for gathering space data, in addition to various small scale, ad-hoc projects, often focused on a single space. While the survey provides useful quantitative and qualitative data (in the form of comments about desired improvements to spaces), conversations with staff at the start of the pilot pointed to questions that were not always answered using survey methodology. Staff questions focused, for example, on whether there was the right mix of collaborative and individual study space both in individual libraries and overall in the system, or if there were underutilized library areas that could be reconfigured to better meet user needs. It was clear that a range of methods was needed to explore these questions more fully, and the authors began to explore how a mixed-methods approach might be implemented system-wide.

To do this, the authors focused on developing space counts and observation protocols for selected spaces. The tool for space counts was a map of the space, which was then used as the basis for visualizing the data (see Figure 1). Counts were conducted during set sampling periods over an entire academic quarter (approximately 10 weeks) by assessment staff, as well as student employees who worked at particular libraries. The pilot began with two smaller spaces within one of our larger libraries to test these tools and explore whether our approach would be scalable across different spaces and sustainable over time. After this initial effort, the tools were refined and then used for increasingly larger and more complex spaces in different libraries. To date, this approach has been tested in six different spaces (four branch libraries of varying sizes, and two distinct spaces within our largest library building). While we have had to develop modifications for larger spaces (carefully targeting our selection of locations to observe in larger libraries, for 
example), the authors have found that the count and observation protocols have been relatively easy to replicate across spaces of different types and sizes.

Figure 1.
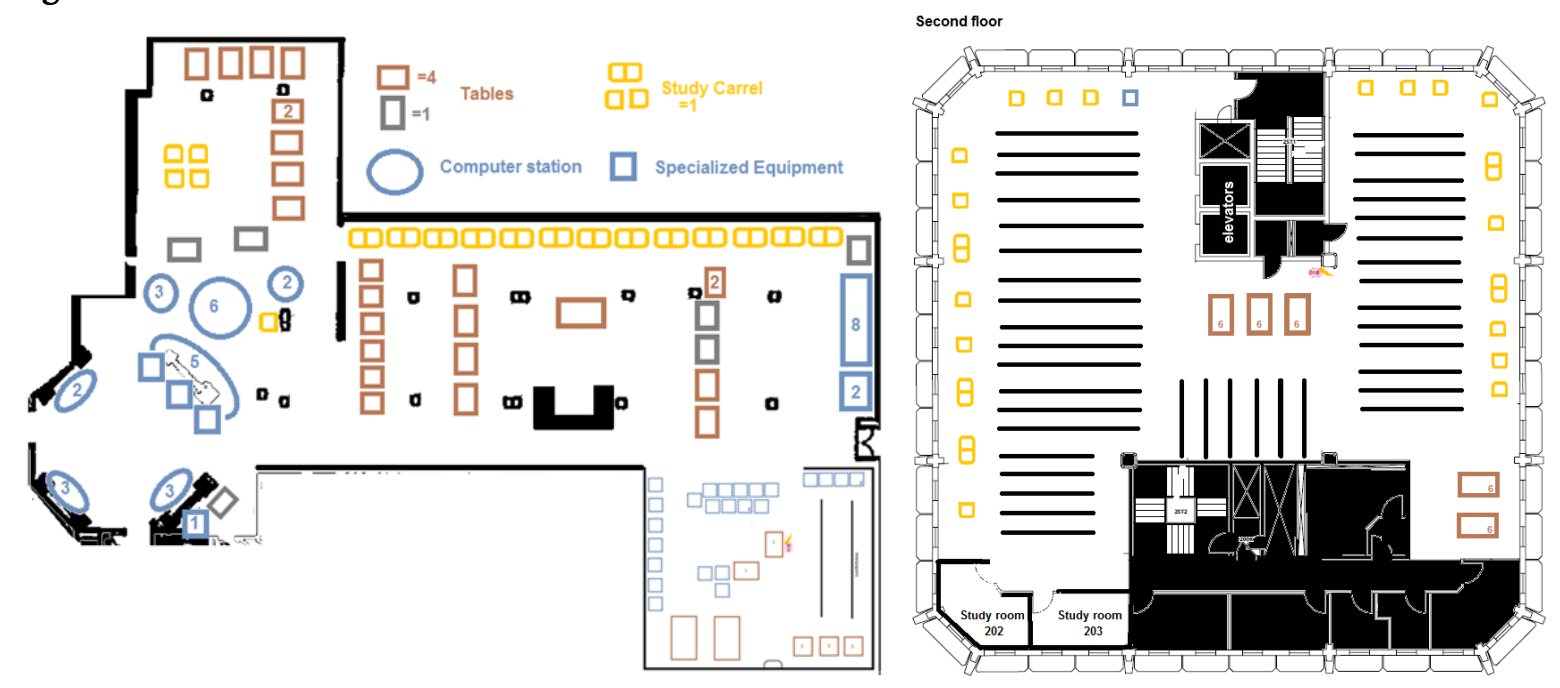

Two maps for space count data collection. Staff ticked off occupied spaces as they walked through the area and assessment staff entered the data in a digital form. Each location indicated on the map has a unique identifier that maps to additional context about the space, e.g., the capacity of the table or type of equipment.

In addition to space counts and observations, the authors also drew upon existing data such as circulation and reference statistics, computer logins, and gate counts. Gathering data using this mixed-methods approach has enabled us to triangulate results and build a more comprehensive picture of space use in different libraries, as well as across the system. This approach spoke directly to staff questions about how specific spaces were being used and provided immediately actionable data for improvements to furniture and seating configurations, as well as signage and placement of key equipment such as charging stations (or the removal of equipment that was not consistently being used).

This approach will also serve us well for longer-term space planning and improvements. Having a snapshot of current use from space counts, for example, will enable us to make better data-informed changes. This will reduce the chance of unintended consequences as we make incremental changes to spaces and respond to one set of needs without considering the whole picture of space use patterns (e.g., if we increase capacity for collaborative space in an underused area, but unintentionally create new space pressures or noise issues as result).

The result of this change to a mixed-methods approach is a set of tools that can be scaled up or down, depending on the size of the library and the particular questions being asked. Having this toolkit means we can now develop a routine schedule for assessments-rotating regularly through all our spaces as needed-so that we can engage in ongoing data gathering. As spaces change, we anticipate updating the data collection tools (such as the maps for space counts) to reflect the most recent configuration.

\section{Focusing on questions and ongoing conversations with staff}

The second area of change for our space assessment program involved surfacing common questions with staff about library spaces. At the start of the project pilot in 2016, and again in early 2018 when revising the latest In Library Use Survey, the authors held a series of discussion sessions with staff across the libraries. In the sessions, we asked staff to brainstorm questions about library spaces and user needs using two brief prompts: 
- What questions do you have about library spaces (how spaces are being used, who is using them)?

- These can include questions not only about the physical spaces themselves, but also questions about the services/resources/technologies provided in the spaces.

- These can be about a specific library or the system as a whole.

- What kind of information about library use would be helpful for improvement? For advocacy/outreach?

As a result, the authors were able to identify a number of common questions across the system, and staff were able to see where their questions were shared. This has provided us with a bank of questions that we are now able to draw upon for more targeted projects either at a single library or across multiple libraries where questions are shared. We are collaboratively reviewing these questions with staff in 2018-19 to decide on which projects to prioritize based on resources and strategic importance.

It is our hope that creating this common set of questions will enable us to move from an ad-hoc approach to space assessment to one that is more collaborative, predictable, and programmatic. This does not preclude library staff from pursuing their own "pop up" assessment projects as needed (informal whiteboard assessments, for example), but it provides a more holistic picture of the questions about our spaces and users that we need to explore together in a more systematic way. In taking this more coordinated approach, we also hope to be better able to surface actionable results from different projects that can be applied to different spaces.

Fostering discussions focused on staff questions about spaces also enabled us to "close the loop" more effectively when reporting assessment results. We mapped results to original staff questions so colleagues could see where their questions were answered and where we might still need to do additional work. The questions that remain unanswered (or new ones arising out of the results) are then added to the bank of shared questions for possible future projects.

\section{Tailored reports and visualizations}

The third area we changed in our approach to space assessment involved how we communicate assessment data to colleagues in order to improve the use of results. Over the course of the pilot, we focused on creating tailored reports and visualizations that combined multiple data sources. These provide a more holistic picture of activity in specific spaces and across the libraries and highlight key messages from users. For example, in reporting the results of the mixed-method space assessment of our Engineering Library conducted in spring 2018, we included quantitative and qualitative data from observations, space counts, the 2018 In Library Use Survey, as well as other library data such as gate counts, circulation, computer logins, and questions asked at service points. Reports also draw upon comments from previous In Library Use surveys and other assessments to highlight where persistent issues still need to be addressed, thus creating a greater sense of urgency about long-standing trends and pain points for users.

The reports that provided a more comprehensive picture of space use were complemented by interactive Tableau dashboards created by one of the authors (the assessment and data visualization librarian); the dashboards are based on space counts and allow staff to explore space and furniture use in different ways: by type of seating or space (e.g., group study rooms, individual study carrels, multi-person tables), by floor of the library, by time of day/day of the week, and by occupancy rate in terms of the capacity for that particular type of space (see Figure 2). 
Figure 2 .

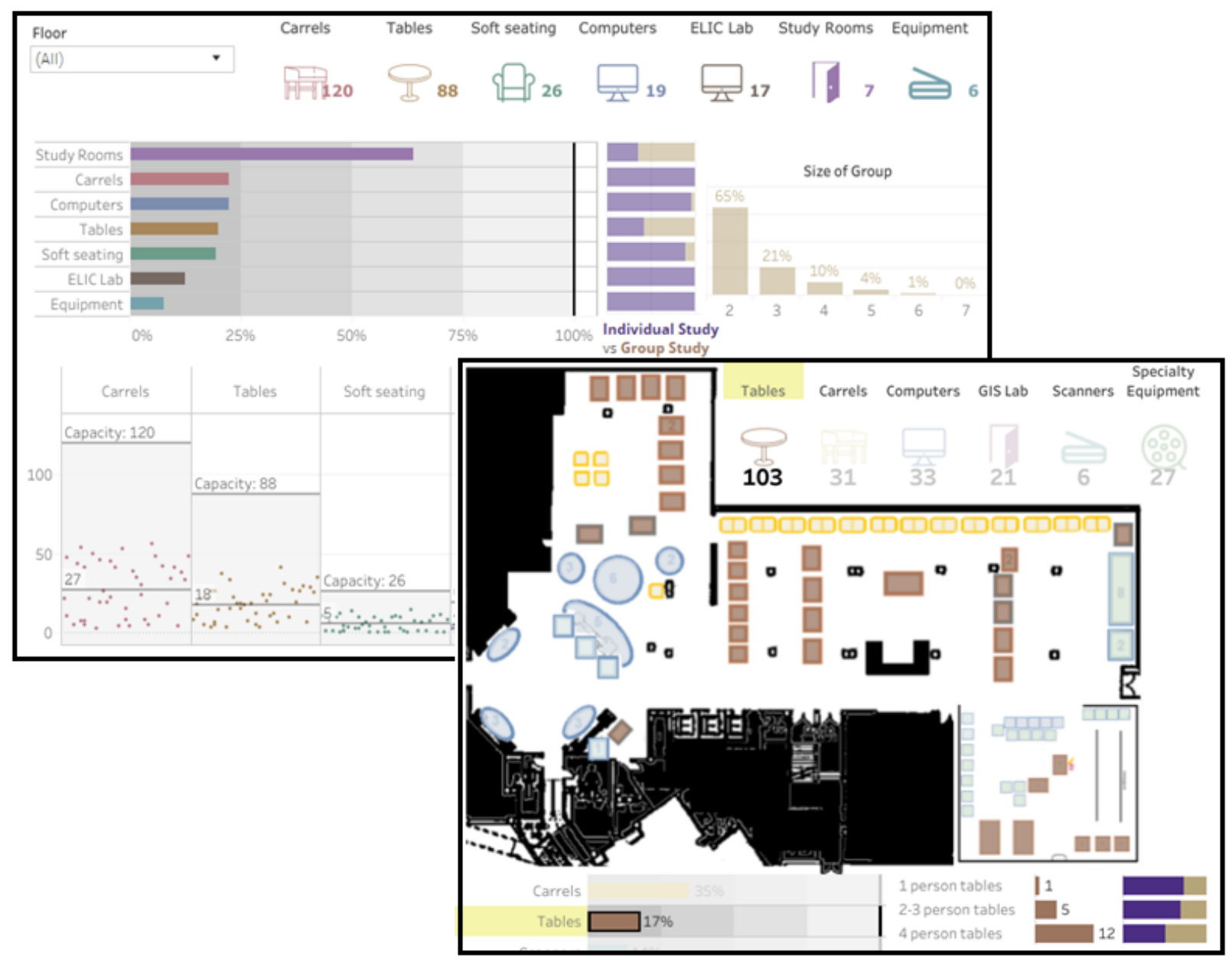

Two space assessment project dashboards in Tableau. Interacting with these dashboards, as shown in the lower right, highlights related information and allows exploration of space count data in additional depth.

While time-intensive to develop, these reports and dashboards now provide the templates we use for all space assessment reporting. Feedback from staff indicates that these have helped staff feel greater ownership of results. In addition, producing this package of results has also reduced the overall time we spend in answering questions about the data. The interactivity provided by the dashboards also allows staff to explore results as additional questions arise, and the dashboards provide some starting, baseline data that staff can use for ongoing decision-making and exploration.

\section{Collaborative analysis, recommendations, decision-making}

A final key change in helping us pilot a more collaborative and sustainable approach to space assessment involved increasing staff involvement in data analysis, decision-making, and communication with users. This change was designed to address the challenge staff often faced in translating results into action from prior space assessments such as the In Library Use Survey. In light of the significant amount of time and resources the UW Libraries invests in developing and implementing space assessments, the authors believed that a truly sustainable approach to our space assessment program needed to be built on a shared commitment to using and communicating results and subsequent improvements. 
In order to help staff make decisions and translate results into concrete actions, we took a variety of approaches during this pilot period. First, we engaged staff in the process of making sense of the data. For mixed-methods space assessments in business, engineering, math, and selected spaces in our largest library (Suzzallo Allen), for example, we provided stakeholders for each project with a draft dashboard and preliminary report with a set of draft observations. Rather than simply presenting the results (in finished reports with recommendations), we engaged staff in conversation about what they saw in the data and whether our observations aligned with their interpretations of the data and their experience in the spaces. We posed questions about what the data meant and what possibilities they saw for action, including their recommendations. We then revised the reports and dashboards accordingly to capture the collective analysis and recommendations. We later returned to staff involved in these projects to see if additional data was needed, if any questions had arisen during the process of acting on data, and what improvements they had made.

Second, the authors held a series of larger group discussions with staff to explore data from the 2018 In Library Use Survey and to generate recommendations collaboratively. We focused these discussions explicitly on how to move from results to action. Our goal was to help staff practice moving from "what is this data saying?" to "what do we do with it?" In addition, we piloted working in partnership with staff members in different libraries to plan these staff sessions. In this approach, we provided some preliminary observations arising out of our survey data analysis, then met with a staff person from that library to identify what seemed actionable based on their experiences of working in the library. While we were able to provide our expertise in data analysis, they provided important context for the data and a clearer vision of those recommendations for improvements that would get wider buy-in from their colleagues. As a result, we were able to highlight the most important results for staff and spend more time talking about what to do with the data during these staff discussions.

Finally, we convened a temporary cross-departmental stakeholder group for our largest library to discuss results and decide who was going to take responsibility for next steps. As discussed previously, uncertainty about who was responsible for acting on results meant that clear (and, in some cases, easily actionable) results often did not lead to changes. Bringing together key stakeholders for the first time allowed everyone to develop a shared understanding of the data and an action plan for implementing changes.

\section{Key takeaways}

This 2016-18 pilot has been a time-intensive effort. While we are continuing to operationalize this new programmatic approach and reflecting on the outcomes of this work, we can offer some observations about what we have learned for those interested in undertaking this kind of programmatic, collaborative work at scale.

One of the most important takeaways from this pilot is the importance of starting small and scaling up; while the first mixed-methods project (as well as the reports and dashboards) took a significant amount of time to develop and implement, we were able to improve the methodology, tools, and processes before moving on to more complex spaces. Taking a gradually scaled approach has enabled us to see what works across different spaces in ways that are sustainable for us and our colleagues. With each subsequent iteration, we have been able to move more rapidly to implement data gathering, analysis, and reporting. In addition, developing a toolkit of tested methods that can be scaled up or down depending on the space and the particular questions being asked means that the methods are adaptable by staff who wish to engage in ongoing and more consistent data gathering in their own libraries.

While taking a mixed-method approach is standard for many space assessment projects, we learned that this is an approach that can be scalable and sustainable for a larger system. The up-front investment of time is significant both to develop the space count tools and observation protocols and to marshal additional relevant library data (such as past survey results, circulation, and computer login data). However, the authors believe this investment has been worthwhile: we now have a system established for gathering and reporting this data in an ongoing way. We found that including data we already collected for reporting 
provided valuable context for the space data and helped legitimize some of our findings as longer-standing trends. Carrying data forward from previous assessment projects helped us highlight ongoing issues for users. As a result, we are engaged in more continuous space improvement for our students and faculty, and also have data available when opportunities arise for more substantial changes and when we need to respond to questions about how our spaces are being used (and the value those spaces provide).

However, using the space counts and observation tools across different libraries of varying sizes-and with staff groups who may have some, but not all, questions in common between their libraries-requires balancing flexibility and consistency, local questions and a system-wide view. In our earlier projects, the authors tended to define categories based on the library's specific needs. For example, one library was only interested in knowing if a study room was occupied, but another was concerned about the size of the groups in the rooms. Our initial data collection between the two was not consistent. Working toward greater consistency in these decisions will help us roll up results more effectively to a system-wide analysis, while maintaining the "on the ground" utility to library staff.

Lastly, collaborating with colleagues has been an important element in terms of a more holistic approach to addressing questions about library spaces and taking action on results. Generating a shared list of spacerelated questions has revealed where coordinated approaches might be taken, which in turn is helping us to establish a schedule for assessments, manage increasing demands on our time, and make ongoing assessment a more routine practice. Likewise, engaging in collaborative discussions focused on decision-making has been key in moving forward with results. However, as we continue this work, we need to explore more effective ways to have these discussions; in the absence of a libraries space committee, for example, the work of coordinating staff conversations across a large system often fell to us, which meant we spent less time engaged in assessment activities and more time facilitating discussions between departments that are sometimes in silos. While this was critical in taking action on assessment results, in the long term, a more coordinated process is needed so we can hand off results to staff more quickly to implement changes.

\section{Next steps}

There are a number of areas we have identified for next steps in our evolving space assessment program. In 2019-20, we hope to roll this approach out more widely to the largest library in the system. This will be our biggest challenge yet in understanding how to scale this mixed-methods approach.

We are also aiming to develop a more coordinated system for tracking improvements and communicating changes to our user communities. In such a large, decentralized library system, changes are often not communicated back to assessment staff, making it challenging to know when and how results are being used. Historically, assessment staff have returned to spaces and noted any changes themselves, but this has not always been consistent; as the pressure of undertaking the next assessment project was felt, following up on changes often fell lower on the list of priorities. The authors are now following up more systematically with staff on changes resulting from these assessment projects and tracking these changes; this will enable us to communicate these improvements more effectively to users.

We are also encouraging staff to communicate with users more routinely on issues arising out of assessment: the Assessment \& Data Visualization Librarian has created a template that staff in a variety of locations can use to share with users what we heard and what we changed. This template is designed to make the process of closing the loop with users more sustainable for library staff. Just as importantly, we are also encouraging staff to communicate with users about why certain changes might not be possible right now, indicating that we have at least heard their feedback.

Most importantly, our longer-term goal for our space assessment program is to center equity and inclusion in consideration of both ongoing improvements and long-term space planning. One element of this is to partner with users more meaningfully in all aspects of the space assessment cycle, from formulating questions, deciding on methods, analyzing data, and shared decision-making. While past space assessments have involved students in design charrettes and focus groups, student involvement in the overall process 
(particularly in shared data analysis and collaborative decision-making) has been limited. A second element involves gaining a better understanding of the ways in which our spaces may not be inclusive of the diverse communities we serve. Our space assessments up to this point reflect those who are already using our libraries, and there are students and faculty who may choose not to work in our spaces because their identities, communities, and ways of working may not be reflected there. ${ }^{6}$ Gaining a better understanding of how we might make our spaces more inclusive for all our students and faculty and how they use available spaces outside the libraries will be a key goal for our space assessment program at the University of Washington Libraries.

-Copyright 2019 Jackie Belanger, Maggie Faber, and Jenna Nobs

\section{Endnotes}

1. Gerke and Teeter, "Counting Heads"; Andrews, Wright, and Raskin, "Library learning spaces," 647-672; Shannon, "Driving the BUS."

2. May and Swabey, "Using and experiencing the academic library."

3. For the purposes of this paper, we are focusing on the libraries at the largest campus, in Seattle, WA. While we are considering how libraries at our Bothell and Tacoma campuses fit into this overall approach, these campuses have different contexts and local needs that are beyond the scope of this paper to discuss.

4. Fournier, Hornby, and Richards, "Active Learning in Odegaard Library."

5. Hiller and Belanger, "User surveys at the University of Washington Libraries."

6. Brook, Ellenwood, and Lazzaro, "In Pursuit of Antiracist Social Justice."

\section{References}

Andrews, C., Wright, S. E., and Raskin, H. "Library Learning Spaces: Investigating Libraries and Investing in Student Feedback." Journal of Library Administration 56, no. 6 (2016): 647-672.

Brook, F., Ellenwood, D., and Lazzaro, A. "In Pursuit of Antiracist Social Justice: Denaturalizing Whiteness in the Academic Library." Library Trends 64, no. 2 (2015): 246-284.

Fournier, J., Hornby, A., and Richards, L. “Active Learning in Odegaard Library: Report on Year 1 of UW's First Active Learning Classrooms.” 2014. http://www.lib.washington.edu/ougl/learning-spaces/activelearning-classrooms/FinalALCReportYearl.pdf.

Gerke, J. and Teeter, K. “Counting Heads: Building Space Assessment into your Library Assessment Plan.” Paper presented at Association of College and Research Libraries: Baltimore, MD, March 24, 2017. http://www.ala.org/acrl/sites/ala.org.acrl/files/content/conferences/confsandpreconfs/2017/Counting Heads.pdf.

Hiller, S. and Belanger, J. "User Surveys at the University of Washington Libraries." In Quality and the Academic Library: Reviewing, Assessing and Enhancing Service Provision, edited by J. Atkinson. Cambridge, MA: Chandos Publishing, 2016, 195-208.

May, F. and Swabey, A. "Using and Experiencing the Academic Library: A Multisite Observational Study of Space and Place.” College \& Research Libraries 76, no. 6 (2015): 771-795.

Shannon, M. "Driving the BUS: A Multimodal Building Use Study and Needs Assessment." In Proceedings of the 2016 Library Assessment Conference, edited by S. Baughman, S. Hiller, K. Monroe, K. \& A. Pappalardo. Washington, DC: Association of Research Libraries, 2016, 80-83. 